\title{
Panorama tecnológico colombiano: una aproximación desde las solicitudes de patentes en Colombia entre los años 2000 y 2018
}

\author{
Colombian technological panorama: an approach from pa-tent applications in \\ Colombia between 2000 and 2018
}

\section{Panorama Tecnológico da Colômbia: uma Abordagem de Pedidos de Patentes na Colômbia entre 2000 e 2018}

\author{
Jesus David Romero-Betancur ${ }^{1}$
}

Recibido: agosto de 2020

Aceptado: diciembre de 2020

Para citar este artículo: Romero-Betancur, J.D. (2021). Panorama tecnológico colombiano: Una aproximación desde las solicitudes de patentes en Colombia entre los años 2000 y 2018. Revista Científica, 40(1), 89-101.

https://doi.org/10.14483/23448350.16929

\section{Resumen}

Un aspecto que permite reconocer el fomento a la innovación en un país se corresponde con la cantidad de patentes solicitadas y otorgadas por este. Entendiendo que, de esta manera, se puede reconocer la dinámica en que el conocimiento se genera y apropia con fines industriales y comerciales. En este contexto, se busca establecer un panorama tecnológico colombiano, basado en las patentes presentadas entre los años 2000 a 2018, según los titulares asociados, así como su origen y naturaleza como persona natural o jurídica (empresas o universidades). Como resultado de este ejercicio se ratifica la percepción generalizada de que en el país se protegen principalmente desarrollos asociados con el sector farmacéutico, encontrando que esto se presenta para el caso de titulares no residentes en el país; mientras que para los residentes el mayor interés se genera por parte de personas naturales sin una especialidad técnica concreta. Por otro lado, se encuentra que las patentes no necesariamente representan un factor de competitividad en el mercado, sino un factor de estatus o distinción para el caso de los nacionales y una herramienta de protección de mercado para los extranjeros. Esto último dado que los objetos de protección por patente en su gran mayoría no se fabrican en el país, sino que solo se comercializan.

Palabras clave: patentes, innovación, gestión de la tecnología, propiedad intelectual, propiedad industrial.

\begin{abstract}
One aspect that allows us to recognize the extent to which innovation is promoted in a country corresponds to the number of patents requested and granted by it, understanding that in this way the dynamics in which knowledge is generated and appropriated for industrial and industrial purposes can be recognized. In this context, it seeks to establish a Colombian technological panorama, based on the patents presented between the years 2000 to 2018, according to the associated holders as well as their origin and nature as a natural or legal person (companies
\end{abstract}

1. Politécnico Internacional. Bogotá, Colombia. jesus.romerob@pi.edu.co 
or universities). As a result of this exercise, the general perception that developments associated with the pharmaceutical sector are mainly protected in the country, finding that this occurs in the case of non-resident holders in the country, while in the case of residents the greater interest is generated by natural persons without a specific technical specialty; On the other hand, it is found that patents do not necessarily represent a factor of competitiveness in the market, but rather a factor of status or distinction in the case of nationals and a tool of market protection for foreigners, the latter especially given that the vast majority of objects of patent protection are not manufactured in the country, they are only marketed.

Keywords: patents, innovation, technology management, intellectual property, industrial property.

\section{Resumo}

Um aspecto que permite reconhecer em que medida a inovação é promovida em um país corresponde ao número de patentes por ele solicitadas e concedidas, entendendo que assim se reconhece a dinâmica em que o conhecimento é gerado e apropriado para fins industriais e industriais. Neste contexto, busca estabelecer um panorama tecnológico colombiano, com base nas patentes apresentadas entre os anos de 2000 a 2018, segundo os titulares associados, bem como sua origem e natureza como pessoa física ou jurídica (empresas ou universidades). Como resultado deste exercício, a percepção generalizada de que os empreendimentos associados ao setor farmacêutico estão principalmente protegidos no país, constatando-se que isso ocorre no caso de titulares não residentes no país, enquanto que no caso de residentes o maior interesse é gerado por pessoas físicas sem especialidade técnica específica; Por outro lado, verifica-se que as patentes não representam necessariamente um fator de competitividade no mercado, mas antes um fator de estatuto ou distinção no caso dos nacionais e um instrumento de proteção do mercado para o estrangeiro, este último especialmente dado que o A grande maioria dos objetos de proteção patentária não é fabricada no país, apenas comercializada.

Palavras-chaves: patentes, inovação, gestão de tecnologia, propriedade intelectual, propriedade industrial.

\section{Introducción}

Con el objetivo de entender las dinámicas de innovación tecnológica en Colombia, el presente trabajo brinda una descripción del comportamiento que ha tenido en el país la presentación, otorgamiento y mantenimiento de patentes durante los dos primeros decenios del nuevo siglo, así como los principales actores relacionados. Este abordaje se sustenta desde la postura de que la protección por patente se otorga sobre una creación novedosa que representa un alto interés técnico o comercial, por lo cual su mantenimiento tiene relación directa con el concepto de innovación.

En un inicio es importante reconocer que las patentes corresponden a un tipo de protección de la propiedad intelectual. Es decir, la protección otorgada a las "creaciones de la mente", expresadas por medio de invenciones, obras literarias y artísticas, así como símbolos, nombres e imágenes utilizados en el comercio (Organización Mundial La Propiedad Intelectual, s. f.). Esta protección se asemeja a cualquier otro derecho de propiedad, permitiendo a su titular gozar de los beneficios derivados de la creación a la vez que busca fomentar desarroIlos intelectuales adicionales. Estos derechos están consagrados en la Declaración Universal de los Derechos Humanos (artículo 27), en la cual se contempla que "toda persona tiene derecho a la protección de los intereses morales y materiales que le correspondan por razón de las producciones científicas, literarias o artísticas de que sea autora" (Organización de las Naciones Unidas, 1948).

La patente como pieza de la propiedad industrial por naturaleza corresponde a un derecho exclusivo otorgado sobre una invención, el cual se concede de forma territorial y temporal, facultando al titular a decidir de qué manera puede ser utilizada por terceros. En un contexto normativo, su origen puede ser datado a 1474 en el marco del estatuto de Venecia, con una finalidad orientada hacia la protección de mercados así como servir de instrumento de política de desarrollo tecnológico y económico de los países (Vidaurreta, 2010). En la actualidad los elementos 
generales de este sistema están regulados por tratados internacionales como el Convenio de París, el Tratado de Cooperación de Patentes (PCT), el Arreglo de Estrasburgo relativo a la Clasificación Internacional de Patentes y, especialmente, el Acuerdo sobre los Aspectos de los Derechos de Propiedad Intelectual relacionados con el Comercio (ADPIC) (Organización Mundial La Propiedad Intelectual, s. f.). Para el caso de Colombia y los demás países de la Comunidad Andina (Bolivia, Perú y Ecuador), el sistema sobre propiedad industrial está regido por la Decisión 486 (Comunidad Andina, 2000).

En términos generales, con una patente pueden protegerse creaciones o invenciones de diversos (casi cualquier) sectores tecnológicos, materializados en productos (compuestos químicos, dispositivos tecnológicos, maquinaria) o procesos (forma de producir un compuesto químico o fabricar un material específico).

Es importante reconocer la marcada diferencia entre la creación de algo nuevo (correspondiente a una novedad) y su aprovechamiento para agregar valor en un contexto específico (configurando una innovación). Entendiendo, además, que esta última se asocia a la idea de mejorar las condiciones de competitividad de las empresas o sectores específicos (Albornoz, 2009; Romero-Betancur y
Pinzón, 2017). La innovación se ha medido como elemento conexo a la investigación y desarrollo (I+D), aun cuando no necesariamente sea desde esta que se gesten las novedades e innovaciones.

La innovación, entendida en sentido amplio, tiene connotaciones sociales, ambientales, económicas, técnicas y tecnológicas. La incorporación de una novedad y su aceptación en alguno de estos ámbitos le da el alcance desde el cual se pueda analizar, a la vez que algo novedoso puede representar innovaciones en diferentes contextos.

La innovación también puede ser entendida desde un contexto tecnológico, cuando supone para la empresa o algún sector de la sociedad la introducción de un cambio técnico en los productos o procesos originado desde la actividad científico-tecnológica (Escorsa-Castells y Valls-Pasola, 1998). Con lo cual, es válido afirmar que esta se relaciona directamente con el aprovechamiento de las patentes otorgadas.

Sin embargo, es importante reconocer que no toda idea novedosa se convierte en patente, aunque sí puede llegar a representar una innovación; de la misma forma que no toda patente alcanza un nivel de apropiación o aprovechamiento tal que la convierta en una innovación tecnológica. En la Figura 1 se presenta un esquema en el que se explica esto.

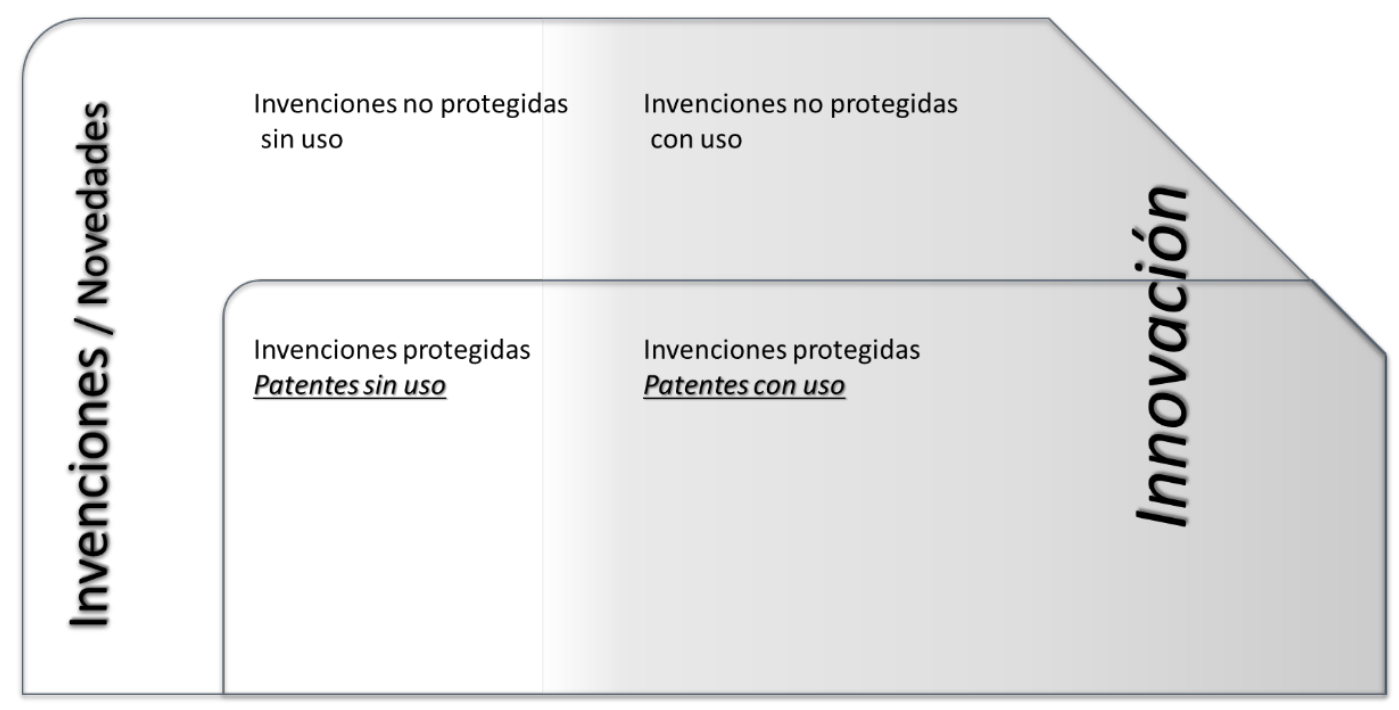

Figura 1. Relación entre invención, patente e innovación.

Fuente: elaboración propia con base en Hernández-Cerdán (2002, p. 22). 
A partir de enfoque, se aprecia cómo las patentes representan un insumo importante para entender las dinámicas propias de la innovación, tanto desde el punto de vista del conocimiento generado que no es aprovechado, como desde esos desarrollos incorporados en cadenas productivas o el mercado en sí. De esta manera se puede establecer, además, que las cifras del total de patentes otorgadas no es necesariamente un indicador de innovación si no se tienen en cuenta en qué medida se está dando el aprovechamiento de estas. Aspecto que emerge al observar del total presentado no solo cuántas patentes se otorgan sino cuántas permanecen en vigor durante el plazo dispuesto.

Por otro lado, es importante tener en cuenta que los ejercicios metodológicos de medición de la innovación se han centrado, principalmente, en los resultados económicos y, más recientemente, sobre las actividades, tomando como base las acciones emprendidas en las empresas así como los recursos destinados para la innovación (Albornoz, 2009). Estas medidas, los indicadores asociados más utilizados o reconocidos y las recomendaciones para el levantamiento de dicha información se encuentran plasmados en la guía para la recolección y reporte de datos de investigación y desarollo experimental, mejor conocido como manual de Frascati (OCDE, 2015) y las directrices para la recolección e interpretación de información relativa a innovación, o manual de Oslo (OCDE, 2018).

En el contexto Latinoamericano, la Red de Indicadores de Ciencia y Tecnología (RICyT), con la principal intención de proveer herramientas para la consolidación de la información relacionada con la actividad empresarial así como las iniciativas y recursos públicos dispuestos para promover la innovación, en el año 2001 publicó el manual de Bogotá: normalización de indicadores de innovación tecnológica en América Latina y el Caribe.

Estos ejercicios propuestos para medir la innovación se basan en encuestas realizadas a empresas, organizaciones gubernamentales e instituciones de educación. Con estas se busca información sobre acciones, gastos e inversión en innovación, así como las capacidades disponibles para esta (Caresani, 2016). En este sentido, las patentes son una herramienta muy importante para el resguardo del conocimiento y su uso sed constituye como un activo fundamental en la toma de decisiones de las empresas para generar innovaciones tecnológicas. Así como reconocer la manera como estas se dan tanto a nivel organizacional como de país.

En el contexto colombiano las patentes como fuente de información han sido abordadas a partir de diferentes enfoques. Desde el mismo sistema de patentes en su conjunto identificando el grado de protección que otorgan los derechos conferidos (Viana Barceló, 2007), como elemento para reconocer las capacidades en I+D del país así como su relación con el desarrollo económico (Sánchez-Blanco, 2006); también han sido abordadas desde un enfoque del desarrollo tecnológico del país (Gómez-Hurtado, 2018) de forma similar a ejercicios llevados a cabo en otros contextos (Rivas y Herruzo, 2000), y desde el enfoque tradicional de estadísticas sobre el comportamiento del sector (Superintendencia de Industria y Comercio, 2015; 2017).

Un ejercicio de alto valor académico en torno a las patentes colombianas fue realizado por Mabel Londoño y Luz Restrepo (2013), quienes retomaron el trabajo del profesor Mayor Mora en su texto "Inventos y patentes en Colombia 1930-2000", un estudio histórico desde un enfoque sociológico alrededor de las patentes de invención en Colombia. Allí se rescatan aspectos como las primeras patentes solicitadas en el país, los orígenes de la reglamentación de la propiedad intelectual en Colombia, así como aspectos de política nacional en esta materia.

Otras miradas desde las que se ha abordado el sistema de patentes y la información asociada a estas ha sido la conformación de redes de cooperación (Agüero-Aguilar, 2017) o la dinámica de publicación internacional de patentes con origen en Colombia (Sánchez et al., 2007), ejercicios que si bien aportan una mirada crítica interesante sobre las dinámicas de la propiedad 
industrial en el país, lo hacen desde puntos de vista aislados y parciales.

De acuerdo con esto, el presente trabajo cobra gran relevancia al proponer una mirada descriptiva del sistema de patentes y los actores participantes en conjunto, abordando la dinámica nacional, por un lado, y reconociendo el comportamiento internacional del conocimiento generado desde el país.

\section{Metodología}

Este trabajo corresponde a un avance de un proyecto de investigación denominado "Innovación tecnológica en Colombia: con base en el estado de las patentes otorgadas entre los años 2000 y 2018", cuyo objetivo fue analizar las dinámicas de innovación tecnológica bajo la hipótesis de que el impacto de las patentes sobre la innovación tecnológica en Colombia es mínimo, pues los principales solicitantes son extranjeros y estos a su vez lo hacen con el fin de frenar la competencia y no dinamizar la economía.

Una primera aproximación que permite la confrontación de esta hipótesis corresponde al estado de las solicitudes de patente en el país del 2000 a 2018, con lo cual se propone abordar el sistema de patentes que va más allá de la simple cuantificación de solicitudes y busca ofrecer un panorama del estado de estas desde el punto de vista de la protección existente. Así como una caracterización general en términos de los solicitantes por tipo y origen geográfico.

De esta forma, en el marco de la investigación desarrollada se propuso abordar como eje de discusión en torno al sistema de patentes colombiano las siguientes preguntas: ¿quiénes y en qué patentan en Colombia? y ¿cuántas patentes otorgadas en Colombia se encuentran actualmente vigentes? y ¿a qué sectores tecnológicos corresponden? Así, el presente texto se centra en dar alcance a la primera pregunta. Aspectos generales de la segunda desde los sectores representados por las empresas con mayor cantidad de solicitudes tramitadas, los sectores tecnológicos declarados en los documentos de patentes, así como el estado de vigencia de las mismas serán temas a abordar en posteriores artículos.

Con el fin de tener información sobre la dinámica de las patentes en Colombia se toma como fuente primaria el registro de la Superintendencia de Industria y Comercio (SIC) en el aplicativo SIPI (sipi.sic.gov.co) ${ }^{2}$. Los datos obtenidos de esta se procesan con el fin de normalizarlos según las variables de interés, entre ellas: nombres de los solicitantes, tipo de solicitantes, país de los solicitantes y año del último estado de la patente. Para esto se genera una matriz de registro en las que se incluye información relacionada con el número de solicitud, nombre de la patente, nombre de los solicitantes (todos los registrados), país de origen de la solicitud, nombre de los inventores, fecha de presentación, estado y clasificación internacional de patentes.

En una revisión de la literatura sobre el análisis de patentes, Abbas (2014) expresa de qué manera la información asociada a estas representa un insumo de gran valor para realizar análisis sobre la novedad de algún desarrollo específico, tendencias tecnológicas, prospectivas tecnológicas, realizar mapeos tecnológicos orientados a determinar vacíos de conocimiento o áreas de interés, identificar competidores tecnológicos, entre otros temas. Además de la representatividad de las patentes en la innovación tecnológica asociada a países o a organizaciones.

A pesar de las limitaciones que puede tener el uso de la información de patentes, el hecho de representar un fuerte eslabón entre los procesos de investigación y desarrollo (I+D) y la innovación, le confiere a las patentes el potencial de servir como indicador tanto de resultado de generación de conocimiento y su aprovechamiento, como de insumo (OCDE, 2009). También permite contar con información estructurada y clara sobre el nivel de madurez de las tecnologías, su relación con otras

2. Los datos tomados de la plataforma SIPI corresponden a los registros públicos al 14 de enero de 2019. 
áreas y las dinámicas propias que se generan en torno a estas.

De esta forma, los datos obtenidos y normalizados del registro de solicitudes de patente presentados en Colombia se usaron para la generación de indicadores con los cuales se esclareció el panorama tecnológico colombiano. Entre ellos se consideran: la cantidad de solicitudes por año, así como el origen del solicitante entre nacional y extranjero y su comportamiento año a año, los registros según el tipo de solicitante entre universidad, empresa o persona natural y el estado de protección en el que se encuentren. En el siguiente apartado se presentará el detalle de esta información y el cálculo de estos indicadores.

\section{Resultados}

\section{Solicitudes de patente en Colombia entre los años 2000 y 2018}

Como resultado inicial se encuentra que durante el periodo de interés se presentaron un total de 36111 solicitudes de protección por patente en Colombia, distribuidas año a año como se muestra en la Figura 2. Se observa que una tendencia creciente en número para todo el periodo; sin embargo, durante los últimos 10 años este comportamiento se ha estabilizado en un valor cercano a las 2200 solicitudes anuales en promedio.

En una aproximación más cercana a los datos se encuentra que de estos 36111 registros, 5897 corresponden a solicitudes presentadas por nacionales (residentes en Colombia), lo que representa un $16 \%$; mientras que por parte de extranjeros se registra el $84 \%$ (30 214). En las Figura 3 y $\underline{4}$ se presenta en detalle esta distribución, así como el comportamiento año a año.

El comportamiento presentado para los datos globales se replica directamente en los registros en cabeza de extranjeros. Sin embargo, el panorama particular para cada tipo de solicitante deja ver que para el caso de los residentes el incremento año a año es sostenido, especialmente en los últimos 10 años; mientras que para los extranjeros durante este mismo periodo de tiempo la tendencia es decreciente, como se muestra en la Figura 4.

Otro aspecto interesante que se puede observar a partir de los registros compilados es el estado actual de las patentes. Para esto, se establecen cuatro posibles estados: concedido, abandonada, negada o en estudio. Cada uno de estos representa una condición en la protección, siendo especialmente importantes para el desarrollo del estudio propuesto aquellas que han sido concedidas; estado en el que se pueden encontrar por presentarse los siguientes casos:

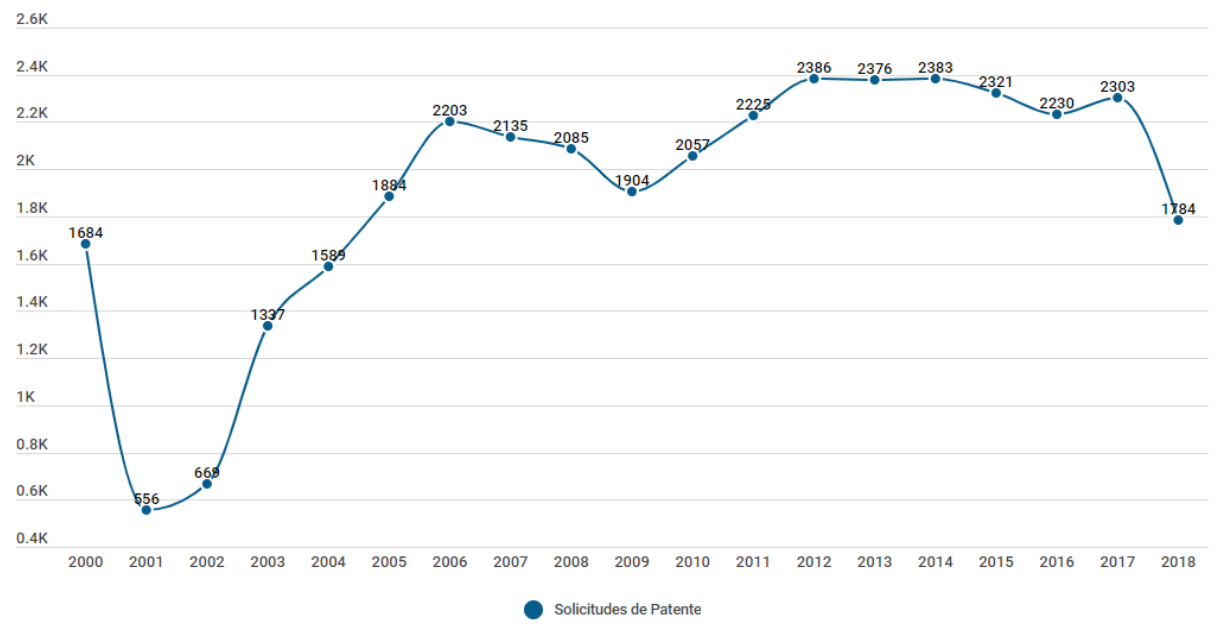

Figura 2. Solicitudes de patente presentadas en Colombia entre los años 2000 y 2018.

Fuente: elaboración propia con base en los datos de la SIC. 


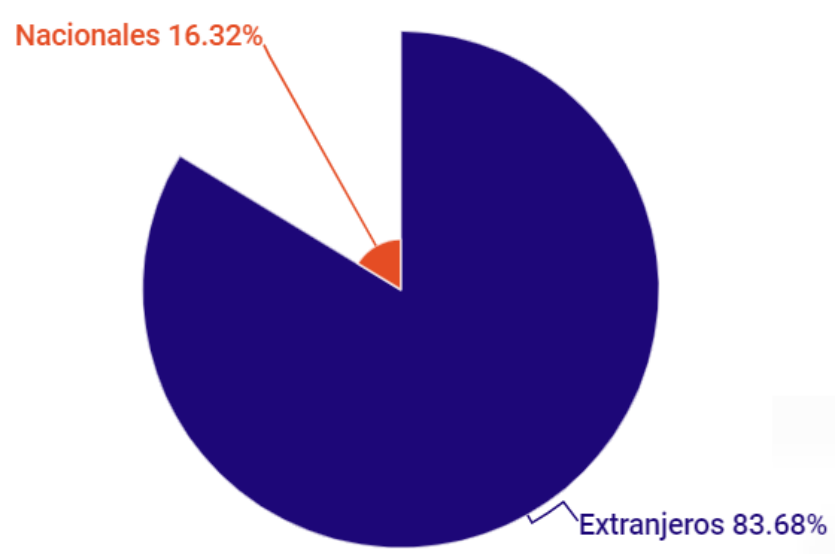

Figura 3. Porcentaje de solicitudes de patente por tipo de solicitante (nacional o extranjero).

Fuente: elaboración propia con base en los datos de la SIC.

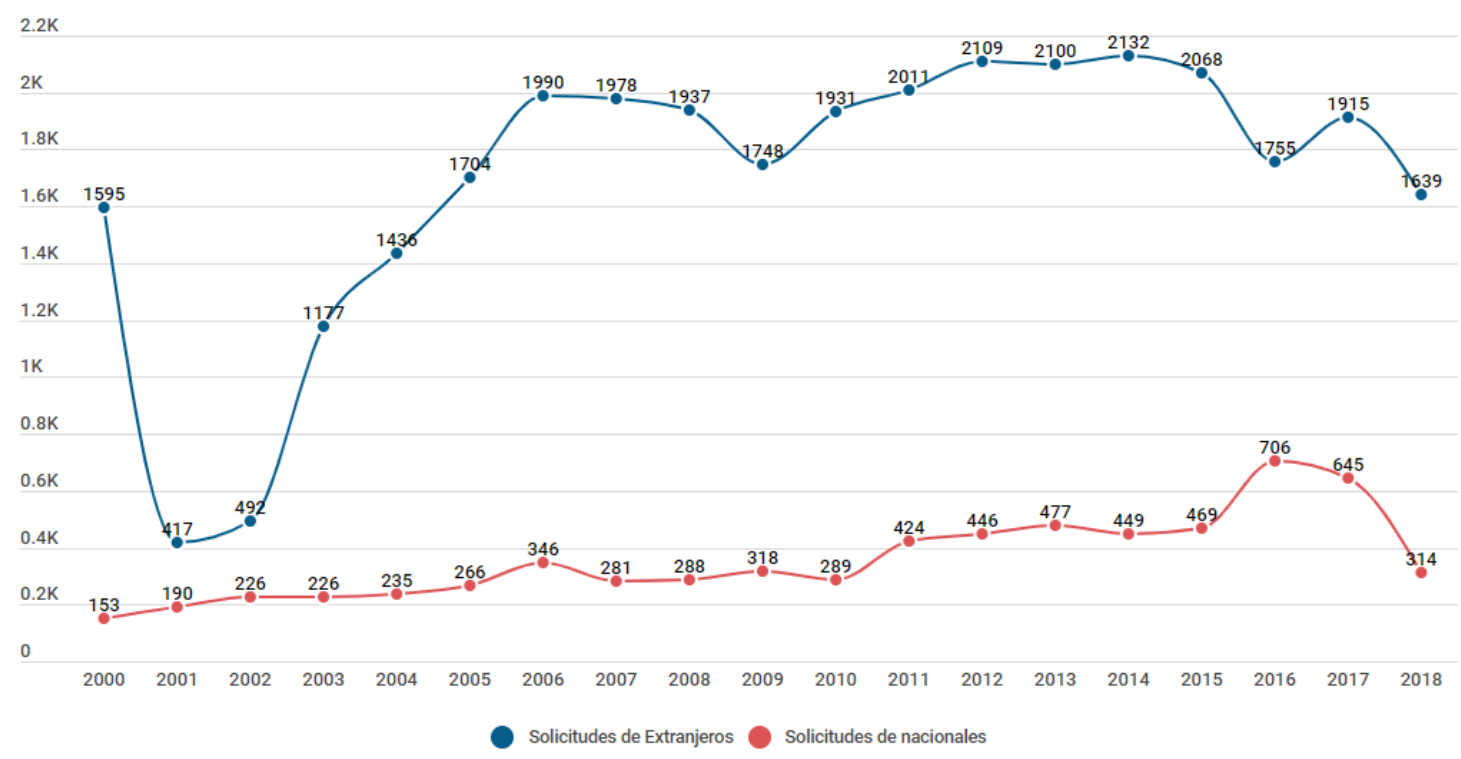

Figura 4. Solicitudes de patente presentadas en Colombia entre los años 2000 y 2018, según tipo de solicitante (nacional o extranjero).

Fuente: elaboración propia con base en los datos de la SIC.

- Activas: solicitudes que han sido aprobadas al cumplir los requisitos de patentabilidad y sobre las cuales se ha realizado el pago correspondiente de la anualidad para su mantenimiento.

- Caducas: patentes otorgadas sobre las que no se realizó el pago de la anualidad correspondiente.

- Dominio público: patentes que finalizaron su periodo de protección.
Teniendo en cuenta que las patentes que se encuentran caducas corresponden a aquellas que fueron otorgadas, pero sobre las cuales dejó de pagarse la anualidad para su mantenimiento, podemos afirmar que el total de patentes concedidas corresponde a aquellas que se encuentran activas más las que han caducado y las que pasaron a dominio público. De acuerdo con esto, más de una tercera parte de las solicitudes presentadas, $38 \%$ 
(13 822), corresponde a aquellas que han sido concedidas; mientras que un número muy cercano (35\%, es decir, 12 534) han sido negadas, como se muestra en la Figura 5. Esto muestra que tan solo poco más de la tercera parte de las invenciones registradas en el país cuentan o han contado con la exclusividad para su explotación.

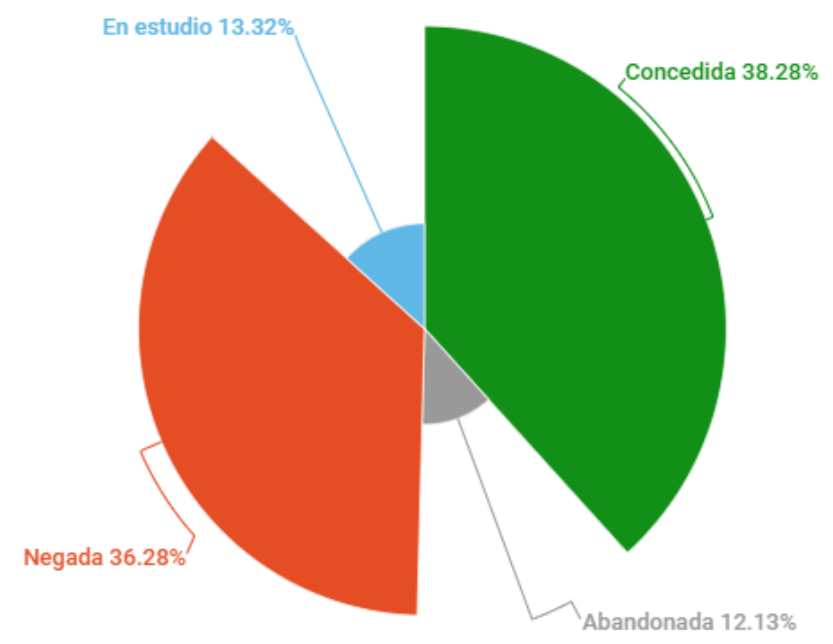

Figura 5. Porcentaje de solicitudes de patente concedidas y negadas.

Fuente: elaboración propia con base en los datos de la SIC.

Por otro lado, en la Figura 6 se observa el comportamiento año a año de esta situación. Se evidencia que en los últimos 10 años la cantidad de protecciones otorgadas a superado a las que han sido negadas, lo que indica un incremento en la efectividad percibida del sistema de patentes colombiano.

\section{Solicitantes de patente en Colombia entre los años 2000 y 2018}

Además de la cantidad de solicitudes, el tipo de solicitante y estado de protección desde una mirada "estática" y "evolutiva" ${ }^{3}$, como parte del panorama tecnológico también es importante realizar una aproximación detallada a las personas (solicitantes). Desde este punto de vista, se encuentra que las 36111 solicitudes de patente presentadas en el periodo de interés han sido registradas por un total de 11146 solicitantes, tanto personas naturales como jurídicas, nacionales y extranjeras.

En la Figura 3 se presentó la distribución de solicitudes según su origen entre nacionales y extranjeros. Allí se observa que el $83 \%$ del total de las solicitudes son presentadas por extranjeros, mientras que como se muestra en la Figura 7, visto

3. Se acuñan estos términos haciendo referencia al análisis general presentado hasta el momento en el cual se expone un panorama general (estático) de las solicitudes de patente totalizadas a la fecha y la discriminación de las solicitudes presentadas entre los años 2000 y 2018 en términos de una mirada evolutiva.

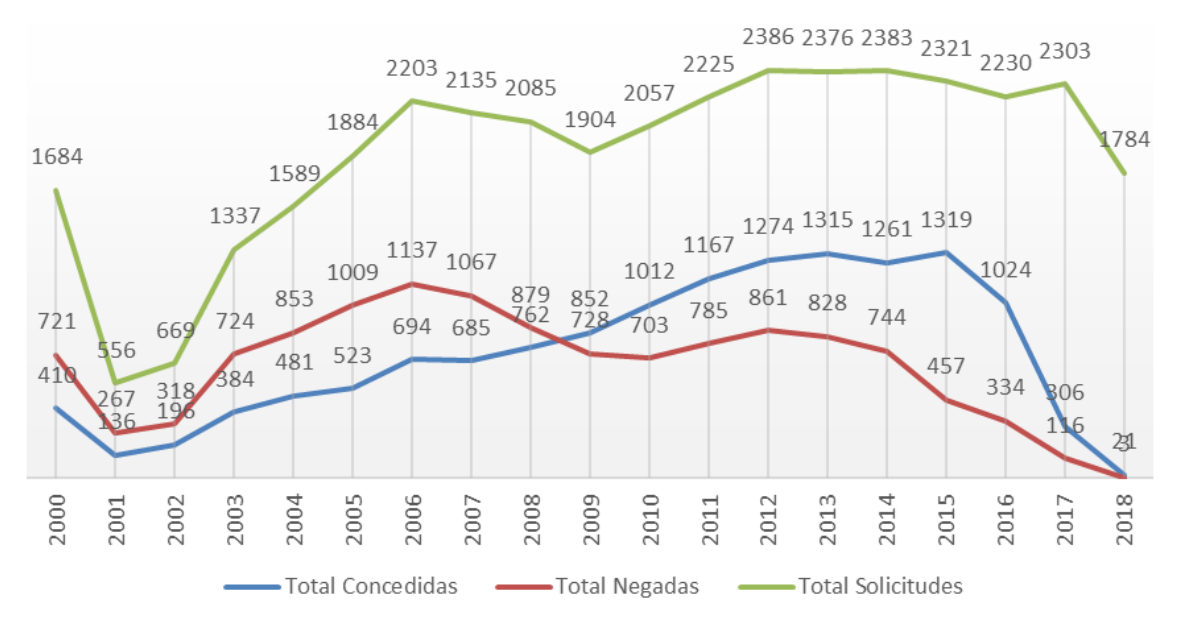

Figura 6. Evolución del otorgamiento y negación de solicitudes de patente en Colombia entre los años 2000 y 2018. Fuente: elaboración propia con base en los datos de la SIC. 
desde el total de solicitantes los extranjeros representan el $65 \%$ de las personas relacionadas en los registros obtenidos. Esto indica que mientras que para los extranjeros se encuentra que por cada solicitante en promedio hay más de cuatro solicitudes de patente, en el caso de los nacionales, la relación es casi 1 a 1, con 1,5 solicitudes por cada solicitante, relación detallada en la Tabla 1.

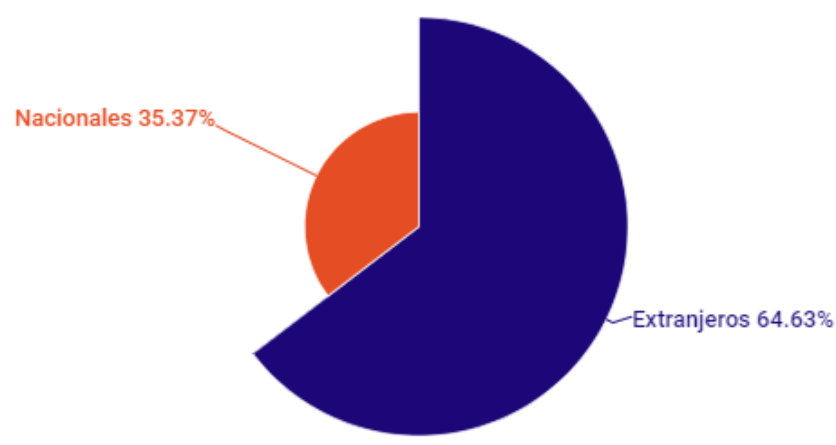

Figura 7. Porcentaje de solicitantes de patente por tipo (nacional/extranjero).

Fuente: elaboración propia con base en los datos de la SIC.

Tabla 1. Relación de cantidad de solicitudes por solicitantes nacional o extranjero.

\begin{tabular}{llll}
\hline $\begin{array}{l}\text { Origen del } \\
\text { solicitante }\end{array}$ & $\begin{array}{c}\text { Total } \\
\text { solicitantes }\end{array}$ & $\begin{array}{c}\text { Total } \\
\text { solicitudes }\end{array}$ & $\begin{array}{c}\text { Relación de } \\
\text { solicitudes por } \\
\text { solicitante }\end{array}$ \\
Extranjero & 7204 & 30214 & 4,2 \\
Nacional & 3942 & 5897 & 1,5 \\
\hline
\end{tabular}

Fuente: elaboración propia con base en los datos de la SIC.

También es importante identificar el tipo de solicitante según su naturaleza. Para esto se establecen tres grupos de interés: empresas, universidades $^{4}$ y personas naturales. Encontrando que la mayoría corresponden al primer grupo con un total de 6962 de los 11146 (62\%), mientras que el

4. En este grupo se consideran las instituciones de educación superior en general, incluyendo las universidades propiamente dichas, así como instituciones universitarias, instituciones tecnológicas e instituciones técnicas profesionales, conforme a la clasificación del Ministerio de Educación Nacional. menor número de solicitantes corresponde a universidades con tan solo el $3 \%$, como se muestra en la Figura 8.

Esta mayor participación de empresas que se encuentra al observar el total de solicitantes no se refleja al detallar el origen de los mismos, bien sean nacionales o extranjeros, como se muestra en la Figura 9. Para el caso de los solicitantes nacionales, la mayor participación se da por las personas naturales, quienes representan cerca del $70 \%$.

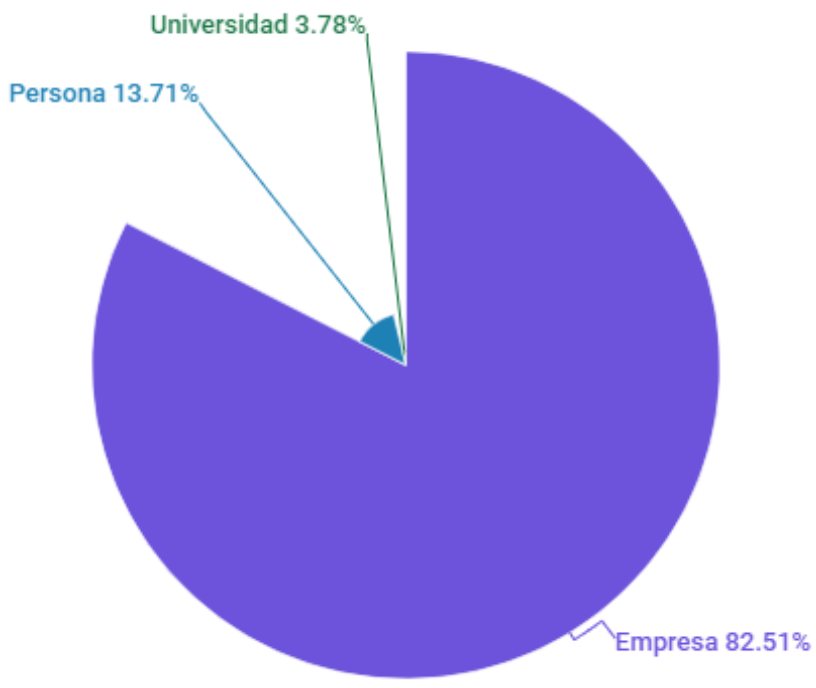

Figura 8. Porcentaje de solicitantes de patente según naturaleza (empresa, universidad o persona natural).

Fuente: elaboración propia con base en los datos de la SIC.

Entendiendo que el solicitante o titular es quien ejerce los derechos patrimoniales o la posibilidad de explotación económica en términos de producir o comercializar el objeto de protección, es importante tener en cuenta que el sector productivo en Colombia tiene un interés menor en acogerse al sistema de patentes como herramienta para resguardar sus desarrollos en comparación con las personas naturales o las mismas empresas extranjeras.

Para el caso de las universidades, se destaca que aun cuando según cifras del Ministerio de Educación Nacional para el 2016 existía un registro de 287 instituciones de educación superior. Siendo este grupo el que más relación tiene con 
la generación y apropiación de conocimiento, encontrar que tan solo una tercera parte del total esté relacionada con patentes sugiere un escaso interés desde este sector por generar nuevo conocimiento o conocimiento tecnológico aplicado a las problemáticas industriales y económicas del país.

En una aproximación más directa a las personas, se destaca que las empresas extranjeras con mayor número de solicitudes de patente en Colombia pertenecen al sector farmacéutico o químico. Entre ellas, las farmacéuticas: Bayer, Novartis, Pfizer, Astrazeneca, Sanofi y Boheringer Ingelheim, así como la empresa Dow Global Technologies del sector agro industrial, lo cual no corresponde con el comportamiento que se tuvo a nivel mundial entre los años 2013 y 2015, periodo en el que la mayoría de las empresas que encabezan las solicitudes de patente en el mundo pertenecen al sector informático y de telecomunicaciones (World InteIlectual Property Organization, 2018, p. 51), entre las que se encuentran compañías como Canon, Samsung, Huawei, ZTE y Qualcomm.

Por otro lado, en el contexto nacional, aunque con un bajo número en relación con el total de registros, los solicitantes con mayor cantidad de registros individuales son universidades. Entre las que se destacan: la Universidad Nacional de Colombia con 108 registros, Eafit con 57, Universidad Militar Nueva Granada con 52, Universidad Industrial de Santander con 50 y Universidad de Antioquia 49. Mientras que la empresa nacional con mayor cantidad de solicitudes es Ecopetrol, que suma 97 en total para el periodo de observación. Vale destacar que en el caso de registros como nacionales también se encuentran multinacionales que aplican al registro desde la filial nacional. En las Figura 10 y 11 se presenta la relación de los 10 principales solicitantes extranjeros y nacionales, respectivamente.

\section{Conclusiones}

Como resultado del proceso de revisión y análisis de las solicitudes de patente presentadas en Colombia entre los años 2000 y 2018 podemos afirmar que durante el periodo de observación el promedio de solicitudes de patente en Colombia se mantuvo cercano a las 2200 por año. Se destaca, además, que durante los últimos 10 años la tendencia de las solicitudes de extranjeros fue decreciente, mientras que las de nacionales presentaron un incremento sostenido, lo que evidencia el

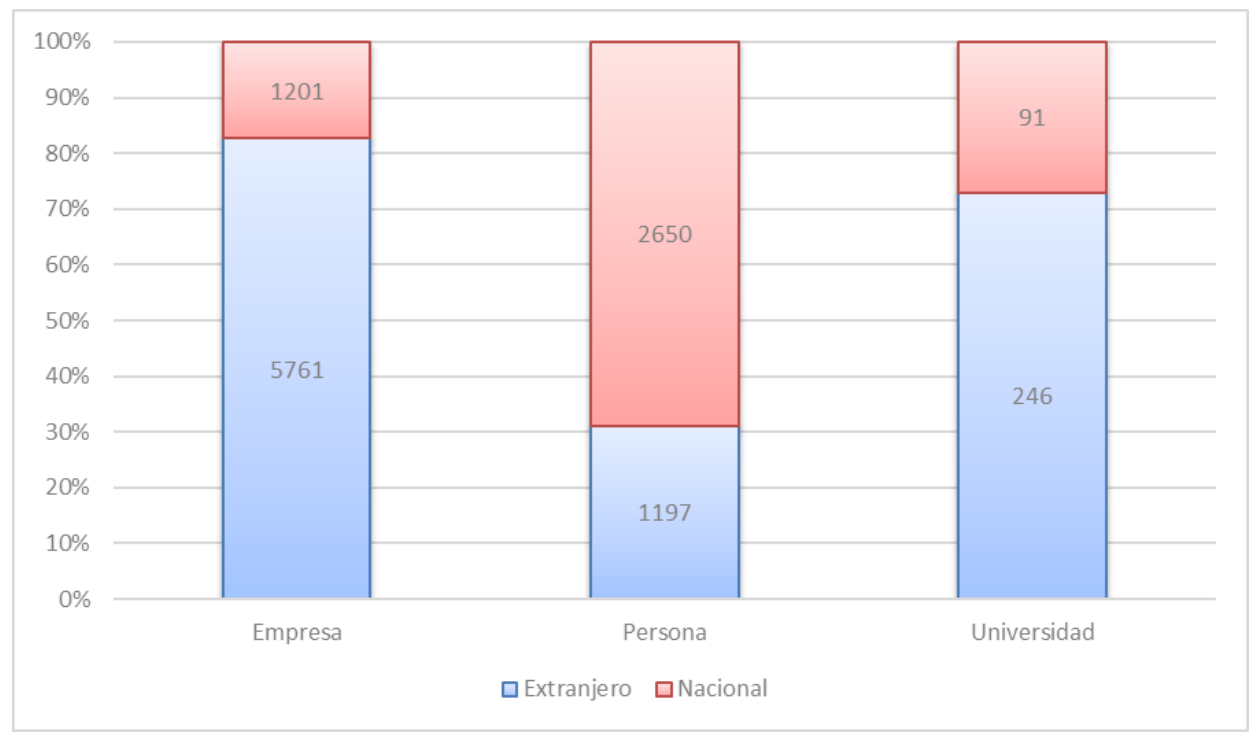

Figura 9. Distribución de los solicitantes según naturaleza y tipo. Fuente: elaboración propia con base en los datos de la SIC. 


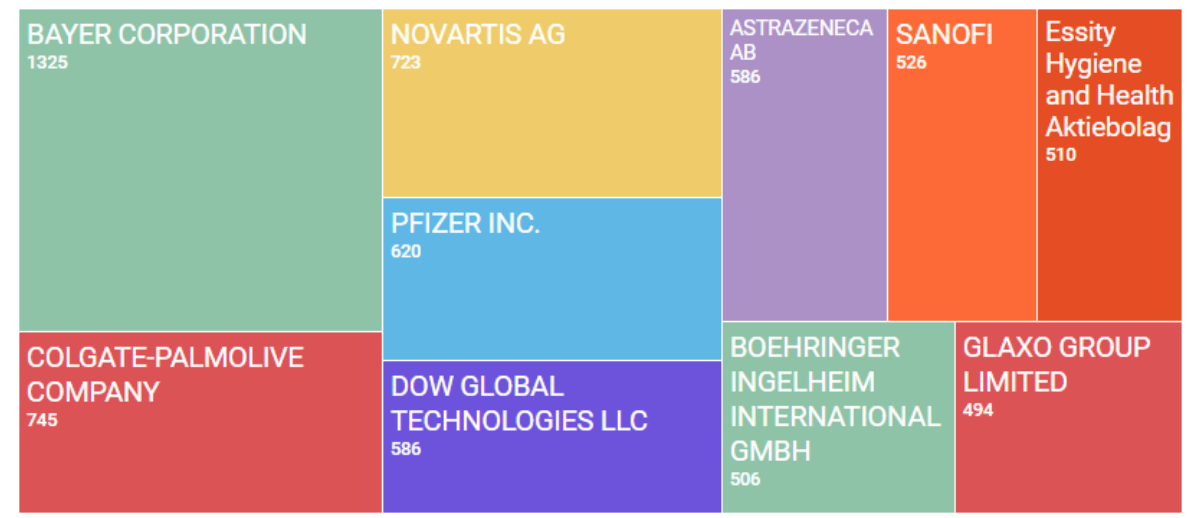

Figura 10. Principales solicitantes extranjeros.

Fuente: elaboración propia con base en los datos de la SIC.

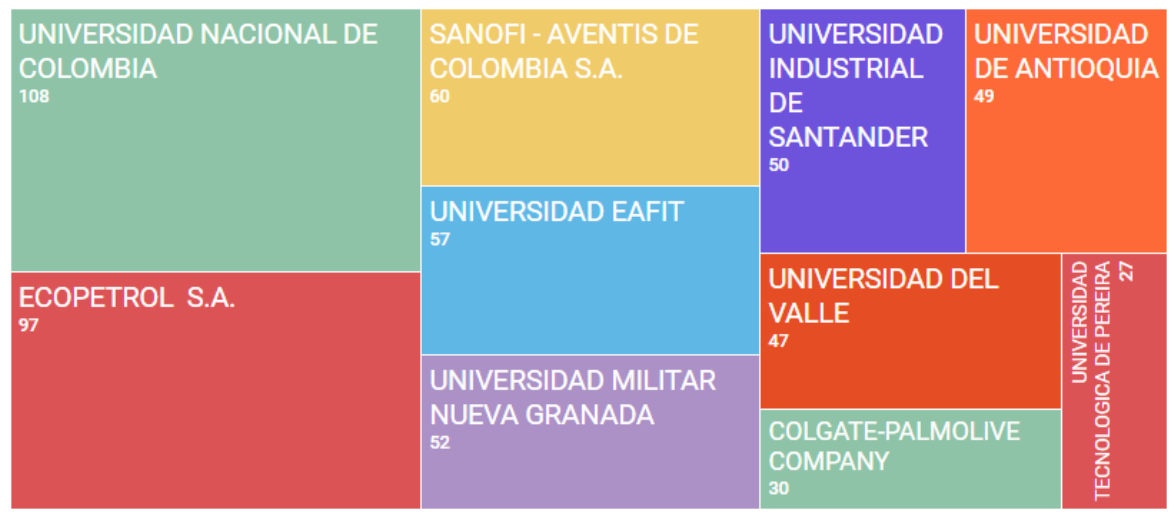

Figura 11. Principales solicitantes nacionales.

Fuente: elaboración propia con base en los datos de la SIC.

esfuerzo nacional por promover el sistema de propiedad industrial mediante programas como el de los Centros de Apoyo a la Tecnología y a la Innovación (Cati $)^{5}$ y los programas de formación ofrecidos desde el Aula de Propiedad Industrial (API) de la SIC.

También se destaca que, al analizar a los solicitantes de patentes agrupados en dos grandes tipos, según su origen y la naturaleza jurídica del mismo, podemos concluir que los solicitantes extranjeros interesados en contar con la protección de sus invenciones en el país corresponden especialmente a empresas del sector farmacéutico. Ello demuestra

5. Véase https://www.sic.gov.co/propiedad-industrial/programa-catipara-instituciones su especial interés por asegurar protección del mercado apoyado en el sistema de patente.

A nivel nacional la mayoría de las solicitudes provienen de inventores independientes, lo que corresponde con el tipo social de inventor colombiano descrito por Mayor Mora como "inventor criollo solitario" (Londoño-Jaramillo y Restrepo-Mejía, 2013), caracterizado por aquellos que se enfocan en distintas direcciones sin una continuidad en sus proyectos y aislado del contexto mundial. Se puede considerar que este tipo de solicitante busca en el sistema de patentes un aliado estratégico para conseguir el resguardo de su capacidad creativa, sin que esto necesariamente garantice un respaldo en la explotación del potencial activo intangible que se configura. 
En relación con el otorgamiento de las patentes, se encuentran dos aspectos de especial importancia. Por un lado, que poco más de la tercera parte de los procesos iniciados ante la SIC resultan en protecciones otorgadas; con lo que se evidencia que el volumen de solicitudes de patente no refleja el nivel de innovación de una economía sino el potencial de innovación existente sujeto a las capacidades con la que cuenten los actores interesados y condiciones del contexto político, económico y social que faciliten su aprovechamiento.

Finalmente, si bien este estudio se centró en el contexto colombiano, es posible indicar que por lo menos en aspectos como el comportamiento de la evolución de las solicitudes, otorgamiento y negación, así como la participación de solicitantes extranjeros y la proporción de estos según su tipo, el comportamiento identificado guarde semejanzas con los demás países de la región, en especial dada la similitud en términos socio económicos que los caracteriza.

Con base en estos resultados, y con el interés de profundizar en el conocimiento obtenido, como futuros temas de investigación se proponen la identificación del grado de importancia en términos tecnológicos y comerciales que representan las patentes otorgadas en el país; así como reconocer las motivaciones para la protección por patente según el actor interesado. En un contexto internacional, aun cuando se puede presumir una similitud en el comportamiento de los países de la región, vale la pena profundizar este análisis con el fin de validar o refutar dicha hipótesis.

\section{Referencias}

Abbas, A., Zhang, L., Khan, S. U. (2014). A literature review on the state-of-the-art in patent analysis. World Patent Information, 37, 3-13. https://doi.org/10.1016/j.wpi.2013.12.006

Agüero-Aguilar, C. E. (2017). Redes de colaboración y producción de patentes en universidades de la Comunidad Andina de Naciones (UCANS) 2005-2015. Revista Española de
Documentación Científica, 40(2), 1-19. https://doi.org/10.3989/redc.2017.2.1401

Albornoz, M. (2009). Res de innovación: las dificultades de un concepto en evolución. Revista Iberoamericana de Ciencia Tecnología y Sociedad, 5(13), 1-12.

Caresani, D. (2016). Antecedentes en la medición de indicadores de la innovación tecnológica. Revista Experiencia Docente, 3(2), 52-58. http://experienciadocente.ecci.edu.co/index. php/experienciadoc/article/view/47

Comunidad Andina (2000). Decisión 486. Régimen común sobre propiedad industrial. www.comunidadandina.org/StaticFiles/DocOf/DEC486.pdf

Escorsa-Castells, P., Valls-Pasola, P. (1998). Innovación tecnológica. En Tecnología e Innovación en la Empresa (pp. 15-44). Ediciones UPC.

Gómez-Hurtado, R. E. (2018). Tendencias de la innovación tecnológica en Colombia 1991-2013 a partir del análisis de patentes. Investigación Bibliotecológica: archivonomía, Bibliotecología e Información, 32(77), 133. https://doi.org/10.22201/ iibi.24488321xe.2018.77.57859

Hernández-Cerdán, J. (2002). Análisis de la innovación a través de las patentes. Universidad Complutense de Madrid.

Londoño-Jaramillo, M., Restrepo-Mejía, L. M. (2013). Patentes: herramientas de innovación. Universidad de Medellín.

Ministerio de Educación Nacional de Colombia (2016). Compendio estadístico educación superior colombiana. Ministerio de Educación Nacional. https://www.mineducacion.gov. co/1759/articles-360739 recurso.pdf \%0Ahttp://www.mineducacion.gov.co/1759/articles-360739 recurso.pdf

OCDE (2009). Manual de estadísticas de patentes de la OCDE (vol. 136). OCDE.

OCDE (2015). Frascati Manual 2015 - Guidelines for collecting and reporting data on research and experimental development. https://doi.org/10.1787/9789264239012-en 
OCDE (2018). Oslo manual 2018 Guidelines for Collecting, Reporting and Using Data on Innovation (4th Editio). https://doi.org/https://doi. org/10.1787/24132764

Organización de la Naciones Unidas (1948). Declaración Universal de Derechos Humanos. Organización de la Naciones Unidas. https://www.ohchr.org/EN/UDHR/Documents/ UDHR Translations/spn.pdf

Organización Mundial de la Propiedad Intelectual (2018). World Intellectual Property Indicators 2018. Organización Mundial de la Propiedad Intelectual.

Organización Mundial de la Propiedad Intelectual (s. f.). Patentes. https://www.wipo.int/patents/es/ Organización Mundial de la Propiedad Intelectual. (s. f.). ¿Qué es la propiedad intelectual? http://www.wipo.int/about-ip/es/

Red Iberoamericana de Indicadores de Ciencia y Tecnología (2001). Manual de Bogotá, Normalización de indicadores de innovación tecnológica en América Latina y el Caribe. Red Iberoamericana de Indicadores de Ciencia y Tecnología.

Rivas, R., Herruzo, A. C. (2000). Las patentes como indicadores de la innovación tecnológica en el sector agrario español y en su industria auxiliar. Patentes como indicadores de la innovación tecnológica en el sector agrario español y en su industria auxiliar. http://www.oepm.es/export/sites/oepm/comun/documentos relacionados/Publicaciones/monografias/patentes/ndicadores.pdf

Romero-Betancur, J. D., Pinzón, F. A. (2017). Innovación, un acercamiento a su concepto, alcance, elementos y capacidades que la generan. Citas, 1, 67-71. http://repository.usta. edu.co/handle/11634/11709

Sánchez, J. M., Medina, J. E., León, A. M. (2007). Publicación internacional de patentes por organizaciones e inventores de origen colombiano. Cuadernos de Economía, 26(47), 247-270.

Sánchez-Blanco, M. C. (2006). La investigación científica y el desarrollo tecnológico (protegido a través del sistema de patentes) y su incidencia sobre el crecimiento económico (Colombia, 1999-2002). https://ciencia.lasalle.edu.co/economia/400

Superintendencia de Industria y Comercio (2015). Estudio Diagnóstico de la propiedad industrial en Colombia. Estudios Económicos Sectoriales, n. ${ }^{\circ} 12$.

Superintendencia de Industria y Comercio (2017). Reporte sobre la información en materia de propiedad intelectual en Colombia. https:// www.sic.gov.co/sites/default/files/files/Proteccion Competencia/Estudios Economicos/ Documentos elaborados Grupo Estudios Economicos/Reporte-informacion-en-materia-de-Propiedad-Intelectual-en-Colombia.pdf

Viana-Barceló, R. (2007). El sistema de patentes en Colombia. Clio América, 1(2), 268-302. https://doi.org/10.21676/23897848.350

Vidaurreta, G. E. (2010). De cómo el criterio utilitarista de justificación primó en los albores del sistema de patentes. Estudio de casos: Inglaterra, Estados Unidos y Francia. Flacso.https:// flascoandes.edu.ec/bitstream/10469/3318/2/ TFLACSO-2011GEV.pdf 